\title{
Addressing the needs of cancer survivors during the COVID-19 pandemic
}

\author{
Larissa Nekhlyudov ${ }^{1}$. Saskia Duijts ${ }^{2,3}$. Shawna V. Hudson ${ }^{4,5} \cdot$ Jennifer M. Jones ${ }^{6,7}$ • Justin Keogh ${ }^{8}$ Brad Love $^{9}$. \\ Maryam Lustberg ${ }^{10} \cdot$ Katherine Clegg Smith $^{11} \cdot$ Amye Tevaarwerk $^{12,13} \cdot$ Xinhua Yu $^{14} \cdot$ Michael Feuerstein $^{15}$
}

Published online: 25 April 2020

(C) Springer Science+Business Media, LLC, part of Springer Nature 2020

\section{Introduction}

The recent COVID-19 pandemic has affected the world and has the potential to disproportionately affect and disrupt the lives of cancer survivors, including those currently in treatment, those who have completed treatment, and those who are now living cancer-free. There are currently over 17 million cancer survivors in the USA [1] and millions more around the world $[2,3]$. Much has been published over the past several decades about the late and long-term effects of cancer treatment, alongside both the challenges and potential solutions to help patients navigate the healthcare system in order to receive high-quality survivorship care $[4,5]$. To date, a number of organizations have provided the cancer survivorship community (both patients and healthcare providers) recommendations pertaining to COVID-19 (Box 1). Unfortunately, at this time, there is limited evidence regarding the impact of COVID-19 on cancer survivors, particularly those who have completed treatment. As the pandemic continues to evolve and scientific evidence emerges, more directed recommendations and guidelines will follow. As editors of the Journal of Cancer Survivorship, the only international peer-reviewed

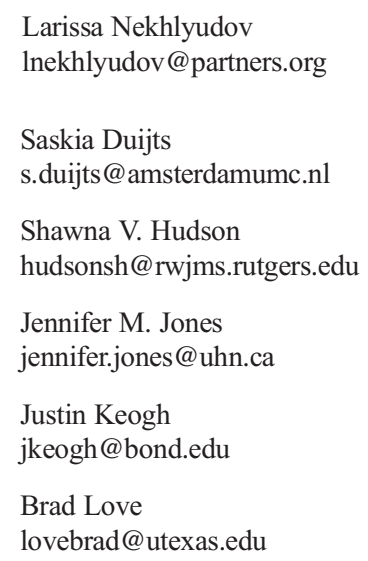

publication dedicated to expanding and disseminating knowledge pertaining directly to this patient population, we wrote this commentary to describe how COVID-19 may impact the physical, psychosocial, and healthcare delivery concerns of cancer survivors. We hope that this information may be helpful in addressing the needs of cancer survivors at the present time and frame the issues that will warrant attention in the future.

\section{Physical and psychosocial issues}

Based on available data, COVID-19 appears to affect people of all ages; however, those who are older and have preexisting medical conditions, including cancer, may be at higher risk for serious medical complications [6, 7]. Whether we consider the more than two-thirds of cancer survivors who are aged 65 and older with pre-existing conditions, those with history of childhood and adolescent/young adult-onset cancer with ongoing late and long-term effects, those who completed bone marrow transplantation, those who remain immunosuppressed post treatment, or those who continue to receive

\author{
Maryam Lustberg \\ Maryam.Lustberg@osumc.edu \\ Katherine Clegg Smith \\ ksmit103@jhu.edu \\ Amye Tevaarwerk \\ at4@medicine.wisc.edu \\ Xinhua Yu \\ xyu2@memphis.edu \\ Michael Feuerstein \\ jcancersurvivorship@gmail.com
}

Extended author information available on the last page of the article 
Box 1 Selected COVID-19 resources for cancer survivors and healthcare providers

\section{General non-cancer support}

World Health Organization

https://www.who.int/emergencies/diseases/novel-coronavirus-2019

Centers for Disease Control and Prevention

https://www.cdc.gov/coronavirus/2019-nCoV/index.html

\section{General cancer-related support}

American Cancer Society

https://www.cancer.org/latest-news/common-questions-about-the-new-coronavirus-outbreak.html

American Society of Clinical Oncology

https://www.asco.org/asco-coronavirus-information

https://www.cancer.net/blog/2020-03/coronavirus-2019-what-people-with-cancer-need-know

American Society of Hematology

https://www.hematology.org/covid-19

Children's Oncology Group

http://survivorshipguidelines.org/

European Society of Medical Oncology

https://www.esmo.org/newsroom/covid-19-and-cancer

National Coalition for Cancer Survivorship

https://www.canceradvocacy.org/blog/coronavirus-cancer-resources-for-survivors/

National Marrow Donor Program/BeTheMatch

https://network.bethematchclinical.org/news/nmdp/be-the-match-response-to-covid-19/

https://bethematch.org/

\section{Selected guidance from non-cancer disease-based organizations}

American College of Cardiology

https://www.acc.org/latest-in-cardiology/features/accs-coronavirus-disease-2019-covid-19-hub\#sort=\%40fcommonsortdate90022\%20descending

American Diabetes Association

https://www.diabetes.org/coronavirus-covid-19

American Thoracic Society

https://www.thoracic.org/professionals/clinical-resources/disease-related-resources/novel-coronavirus.php

\section{Psychological and social support}

Cancer Support Community

https://www.cancersupportcommunity.org/blog/2020/03/what-cancer-patients-survivors-and-caregivers-need

CancerCare

https://www.cancercare.org/

GW Cancer Center

https://smhs.gwu.edu/cancercontroltap/resources/advancing-patient-centered-cancer-survivorship-care-toolkit

\section{Exercise support}

American Society of Sports Medicine

https://www.acsm.org/read-research/newsroom/news-releases/news-detail/2020/03/16/staying-physically-active-during-covid-19-pandemic

\section{Employment support}

\section{Career and Cancer}

https://www.cancerandcareers.org/

\section{Communication about COVID-19}

Center to Advance Palliative Care

https://www.capc.org/toolkits/covid-19-response-resources/

Vital Talk

https://www.vitaltalk.org/guides/covid-19-communication-skills/

Note: Resources listed here are mainly those in the USA. We encourage the readers to seek guidance from their local, regional, and/or national sources. Further, given the fluidity of the pandemic, the links provided may also change 
immunosuppressive therapy, cancer survivors are at risk for COVID-19 and its potential complications. As the COVID-19 pandemic evolves, there is currently no evidence to suggest that different precautions should be recommended for cancer survivors compared to the recommendations by the World Health Organization (WHO) for the general population (e.g., physical distancing, washing hands with soap and water, and avoiding touching the face) [8]. At this time, cancer survivors should adhere to general protocols available through their communities (if not available, the WHO). However, it is important that clinicians communicate with their patients about their individual risks, taking into account their underlying health conditions, and counsel them about "if, when, and where" to seek medical attention.

In addition to the implications of COVID-19 on cancer survivors on their physical well-being, the psychosocial impact of the pandemic may also disproportionately affect this population. Cancer survivors have been previously described as some having unique emotional needs related to anxiety, depression, fear of recurrences as well as family, interpersonal, employment, and financial strain [9-11]. While some survivors may feel that they have "been through worse," for others, this pandemic may raise fears related to prior traumatic health experiences (for example, being hospitalized during treatment and being on isolation due to neutropenia). The recommendations from the WHO, country and state-based governments, and other public health experts have been advising "social distancing" - which is more accurately described as "physical distancing" (e.g., shutting down of in-person support groups, avoiding large group gatherings in places of worship and other community organizations, and distancing families and social circles) which may present particular challenges for survivors for whom such contact may be critical. It is important that clinicians are aware of the negative consequences of such distancing for those already at risk for distress. Online cancer support groups may be helpful for those feeling isolated and distressed [12].

While there is no existing evidence at this time, it is possible that the stress related to this pandemic may exacerbate other treatment-related effects reported among cancer survivors, including cognitive problems, such as memory loss and slowing of information processing and problem solving. This is particularly challenging as changes have been rapid and ongoing. The COVID-19-driven closures of non-essential services and redirection of healthcare resources may also increase anxiety and confusion among cancer survivors. Survivors who are receiving ongoing cancer treatment may struggle with tough decisions about the availability of treatment, imaging, and other services while perhaps also feeling anxious about the potential risks of getting infected with COVID-19 in clinical facilities. Due to ongoing concerns about the availability of healthcare resources, such as ventilators, cancer survivors may fear for possible prioritization that may take place should such resources become too scarce [13]. Directly acknowledging these concerns and anxieties is important. Use of eHealth interventions may be useful in addressing specific psychosocial and physical symptoms in cancer survivors [14, 15]. Publically available mobile technology (i.e., Facetime, Skype, and Zoom) may promote alternatives to social distancing and provide informational connection despite the physical distance.

As some survivors may have the added responsibilities such as balancing working from home and caring for children or other family members, it is important to emphasize that this is not the time to be "super productive" (e.g., home school, organize the home, and write a novel). While starting a new routine can be a challenge, this may, however, be an opportunity to continue to support cancer survivors to focus on healthy behaviors, such as engaging in physical activity, reducing sedentary time, consuming a healthy diet, and obtaining sufficient sleep. Distanced-based interventions including eHealth technology has shown promise in supporting cancer survivors to increase their physical activity $[16,17]$ and may provide survivors a mechanism for empowerment and control during this pandemic.

Survivors may also be at particular risk for financial impact related to COVID-19. As the pandemic takes its toll on the global economy, working-age cancer survivors might lose their job and, in some parts of the world, possibly their health insurance. Being able to work has been shown to affect the quality of life of cancer survivors as having a job contributes to feelings of normalcy, resilience, and meaning [18]. Those individuals who are self-employed or work for small employers may also face unique short- and long-term uncertainties. Moreover, research has shown that those with a history of cancer have more problems in finding a new job after job loss compared to the general healthy population [19]. Survivors whose employment has not been affected by the pandemic or who may be considered by their local jurisdictions as "essential" employees have to weigh the benefits of work with potential increased exposure depending on their occupational risks. As governments continue to explore methods to stimulate the economy and support those in need, it is critical that there is recognition of the challenges that are likely going to be faced by cancer survivors.

\section{Cancer survivorship care delivery}

The delivery of high-quality survivorship care can be challenging, including access to multi-specialty and multidisciplinary healthcare professionals with the necessary expertise. As the COVID-19 pandemic evolves, many cancer centers may need to prioritize their resources to provide care to those who are newly diagnosed and/or are in active treatment [20]. Efforts are also being made to reduce the exposure of 
other cancer survivors to potential COVID-19 by moving inperson visits to telemedicine or otherwise redirect non-acute care. Follow-up visits and surveillance imaging may be put on hold or made into "virtual appointments" with physical examinations deferred. Likewise, primary care, specialty care, and other healthcare facilities may be either closed for in-person visits or drastically scaled back. As discussed above, this disruption may increase anxiety for cancer survivors, and though there is no current evidence for negative long-term consequences (such as delayed diagnoses, worsened health outcomes), these should be considered. Though challenging, it is imperative that clinicians continue to offer ongoing contact with these patients, review their symptoms, and provide reassurance as needed through virtual health platforms, including the existing mobile applications that are currently available [21]. There may also be an opportunity to expand remote monitoring of patient-reported outcomes [22-24] and pave the way for survivors to become more engaged and activated [25]. Clinicians can help by acknowledging and normalizing increased levels of anxiety and fear of recurrence and providing appropriate psychosocial referrals when severe [26].

Notwithstanding the potential benefits of technology in this setting, it is important to recognize that the current crisis may pose further challenges to communities that have under "normal circumstances" faced barriers to healthcare access, such as those living in rural environments, as well those low-income, uninsured or underinsured, racial/ethnic minority, and sexual minority medically underserved cancer survivors $[27$, $28]$. Whereas for some populations, rapidly developing implementation of telemedicine options may provide new ways to access care and help to break down existing barriers, not all will be served by such measures.

COVID-19 has already altered the face of survivorship care. However, it is important to note that it may also lead to innovations in healthcare delivery. Previously, despite its great potential, telemedicine faced many barriers to successful implementation, ranging from reimbursement, regulatory (e.g., state restrictions on provider licensing) to patient or provider attitudes (e.g., preferring a face-to-face visit or aversion to "new" technology). During the COVID-19 crisis, telemedicine approaches are being promoted for rapid deployment in most populations. Strategies range from simple patient health portal messages to relieve triage phone lines, asynchronous econsultation, to telephone- or video-based virtual visits. Given the need for quick action, certain restrictions have been relaxed (for example in the USA, those surrounding reimbursement and licensing have been temporarily suspended). Despite this, many challenges remain, including (1) training survivors and healthcare providers to participate in telemedicine visits in the midst of a pandemic, (2) unequal broadband and cellular access, and (3) relatively low levels of familiarity with patient health portals on the part of many survivors and others [29]. This pandemic presents a unique opportunity to test the feasibility of applying telemedicine procedures for cancer survivors on a much larger and more rapidly deployed scale than previously anticipated. It is possible that this medium will continue to expand and allow greater access to expertise and promote increased collaboration among healthcare providers that has positive impacts that extend beyond this moment of crisis. However, the potential downstream effects of deferred and/or delayed in-person services related to this pandemic will need to be measured and appropriately addressed. In order to do so, there must be systematic efforts in place designed to capture data related to these processes and outcomes.

\section{Conclusion}

In summary, cancer survivors face challenges under the "best" of day-to-day circumstances. We are currently experiencing an unprecedented crisis that has affected all aspects of the global community. We have compiled a list of resources that can be shared with clinicians and patients (Box 1). While there is uncertainty in how this pandemic will evolve, what healthcare issues will emerge, and how technology will advance, this editorial was written as a reminder that all healthcare providers should remember that many cancer survivors need tailored, patient-centered care during these trying times. It will be important that the cancer survivorship community continue to collect and share evidence and resources that may be of benefit for this population at risk today and as the pandemic eventually resolves.

\section{Compliance with ethical standards}

Conflict of interest The authors declare that they have no conflict of interest.

\section{References}

1. Miller KD, Nogueira L, Mariotto AB, Rowland JH, Yabroff KR, Alfano CM, et al. Cancer treatment and survivorship statistics, 2019. CA Cancer J Clin. 2019;69:363-85.

2. The Cancer Atlas. https://canceratlas.cancer.org/the-burden/cancersurvivorship/. Accessed March 31, 2020.

3. Global Cancer Observatory. http://gco.iarc.fr/. Accessed March 31, 2020.

4. Kline RM, Arora NK, Bradley CJ, Brauer ER, Graves DL, Lunsford NB, et al. Long-term survivorship care after cancer treatment-summary of a 2017 National Cancer Policy Forum workshop. J Natl Cancer Inst. 2018;110:1300-10.

5. Nekhlyudov L, Mollica MA, Jacobsen PB, Mayer DK, Shulman LN, Geiger AM. Developing a quality of cancer survivorship care framework: implications for clinical care, research, and policy. J Natl Cancer Inst. 2019;111:1120-30. 
6. Yu J, Ouyang W, Chua MLK, Xie C. SARS-CoV-2 transmission in patients with cancer at a tertiary care hospital in Wuhan, China. JAMA Oncol. 2020. https://doi.org/10.1001/jamaoncol.2020.0980.

7. Liang W, Guan W, Chen R, Wang W, Li J, Xu K, et al. Cancer patients in SARS-CoV-2 infection: a nationwide analysis in China. Lancet Oncol. 2020;21:335-7.

8. World Health Organization Coronavirus disease (COVID-19) pandemic. https://www.who.int/emergencies/diseases/novelcoronavirus-2019. Accessed March 31, 2020.

9. Reed SC, Bell JF, Miglioretti DL, Nekhlyudov L, Fairman N, Joseph JG. Fear of cancer recurrence and associations with mental health status and individual characteristics among cancer survivors: findings from a nationally representative sample. J Psychosoc Oncol. 2020;38:125-42.

10. Carrera PM, Kantarjian HM, Blinder VS. The financial burden and distress of patients with cancer: understanding and stepping-up action on the financial toxicity of cancer treatment. CA Cancer J Clin. 2018;68:153-65.

11. Aaronson NK, Mattioli V, Minton O, Weis J, Johansen C, Dalton $\mathrm{SO}$, et al. Beyond treatment - psychosocial and behavioural issues in cancer survivorship research and practice. EJC Suppl. 2014;12: $54-64$.

12. American Cancer Society. https://www.cancer.org/treatment/ support-programs-and-services/online-communities.html. Accessed March 31, 2020.

13. Daniel Sokol: The life and death decisions of COVID-19. https:// blogs.bmj.com/bmj/2020/03/20/daniel-sokol-the-life-and-deathdecisions-of-covid-19/. Accessed March 31, 2020.

14. Agboola SO, Ju W, Elfiky A, Kvedar JC, Jethwani K. The effect of technology-based interventions on pain, depression, and quality of life in patients with cancer: a systematic review of randomized controlled trials. J Med Internet Res. 2015;17:e65.

15. Harris J, Cheevers K, Armes J. The emerging role of digital health in monitoring and supporting people living with cancer and the consequences of its treatments. Curr Opin Support Palliat Care. 2018;12:268-75.

16. Dorri S, Asadi F, Olfatbakhsh A, Kazemi A. A systematic review of electronic health (eHealth) interventions to improve physical activity in patients with breast cancer. Breast Cancer. 2020;27:25-46.

17. Haberlin C, O'Dwyer T, Mockler D, Moran J, O'Donnell DM, Broderick $\mathrm{J}$. The use of eHealth to promote physical activity in cancer survivors: a systematic review. Support Care Cancer. 2018;26:3323-36.

18. de Boer AG, Torp S, Popa A, et al. Long-term work retention after treatment for cancer: a systematic review and meta-analysis. J
Cancer Surviv. 2020:1-16. https://doi.org/10.1007/s11764-02000862-2.

19. van Egmond MP, Duijts SFA, Loyen A, Vermeulen SJ, van der Beek AJ, Anema JR. Barriers and facilitators for return to work in cancer survivors with job loss experience: a focus group study. Eur J Cancer Care (Engl). 2017;26. https://doi.org/10.1111/ecc.12420.

20. Kutikov A, Weinberg DS, Edelman MJ, Horwitz EM, Uzzo RG, Fisher RI. A war on two fronts: cancer care in the time of COVID19. Ann Intern Med. 2020. https://doi.org/10.7326/M20-1133.

21. Garg S, Williams NL, Ip A, Dicker AP. Clinical integration of digital solutions in health care: an overview of the current landscape of digital technologies in cancer care. JCO Clin Cancer Inform. 2018;2:1-9.

22. Basch E, Deal AM, Kris MG, et al. Symptom monitoring with patient-reported outcomes during routine cancer treatment: a randomized controlled trial. J Clin Oncol. 2016;34:557-65.

23. Denis F, Basch E, Septans AL, Bennouna J, Urban T, Dueck AC, et al. Two-year survival comparing web-based symptom monitoring vs routine surveillance following treatment for lung cancer. JAMA. 2019;321:306-7.

24. Katz MS. Cancer care in the time of COVID-19. https://connection. asco.org/blogs/cancer-care-time-covid-19. Accessed March 31, 2020.

25. Calvert MJ, O'Connor DJ, Basch EM. Harnessing the patient voice in real-world evidence: the essential role of patient-reported outcomes. Nat Rev Drug Discov. 2019;18:731-2.

26. Butow P, Sharp L, Thewes B, Turner J, Gilchrist J, Beith J. Fear of cancer recurrence: a practical guide for clinicians. Oncology (Williston Park). 2018;32(1):32-8.

27. Kent EE, Wheldon CW, Smith AW, Srinivasan S, Geiger AM. Care delivery, patient experiences, and health outcomes among sexual and gender minority patients with cancer and survivors: a scoping review. Cancer. 2019;125:4371-9.

28. O'Malley DM, Tsui J, Davis SN, Hudson SV. Disparities. In: Feuerstein M, Nekhlyudov L, editors. Handbook of cancer survivorship. New York: Springer Science+Business Media; 2018. p. 73-89.

29. ONC Data Brief No. 50. Access and use of electronic health information by individuals with cancer: 2017-2018. https://www. healthit.gov/sites/default/files/page/2020-01/ 2018HINTSCancerDB.pdf. Accessed March 31, 2020.

Publisher's note Springer Nature remains neutral with regard to jurisdictional claims in published maps and institutional affiliations. 


\section{Affiliations}

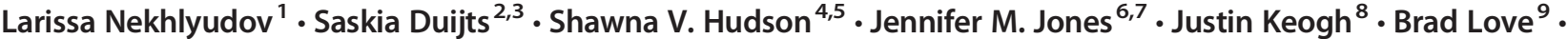 Maryam Lustberg $^{10} \cdot$ Katherine Clegg Smith $^{11} \cdot$ Amye Tevaarwerk $^{12,13} \cdot$ Xinhua Yu $^{14} \cdot$ Michael Feuerstein $^{15}$}

1 Department of Medicine, Brigham \& Women's Hospital, Harvard Medical School, Boston, MA, USA

2 Department of Public and Occupational Health, Amsterdam UMC Vrije Universiteit Amsterdam, Amsterdam, The Netherlands

3 Netherlands Comprehensive Cancer Organization (IKNL), Utrecht, The Netherlands

4 Department of Family Medicine and Community Health, Rutgers Robert Wood Johnson Medical School, Brunswick, NJ, USA

5 Rutgers Cancer Institute of New Jersey, New Brunswick, NJ, USA

6 Cancer Rehabilitation and Survivorship Program, Princess Margaret Cancer Centre, Toronto, Canada

Department of Psychiatry, University of Toronto, Toronto, Canada

8 Faculty of Health Sciences and Medicine, Bond University, Gold Coast, Australia
9 Center for Health Communication, Moody College of Communication and Dell Medical School, University of Texas at Austin, Austin, TX, USA

10 The Ohio State Comprehensive Cancer Center, Columbus, OH, USA

11 Department of Health, Behavior and Society, Johns Hopkins Bloomberg School of Public Health, Baltimore, MD, USA

12 Hematology/Oncology, University of Wisconsin-Madison, Madison, WI, USA

13 Carbone Cancer Center, Madison, WI, USA

14 Division of Epidemiology, Biostatistics and Environmental Health, University of Memphis, Memphis, TN, USA

15 The Journal of Cancer Survivorship, Gaithersburg, MD, USA 